\title{
System-Level Analysis of Closed-Loop Power Control in the Uplink of DS-CDMA Cellular Networks
}

\author{
Luis Mendo and José M. Hernando, Member, IEEE
}

\begin{abstract}
A system-level model for power control in the uplink of direct-sequence code-division multiple access (DS-CDMA) cellular networks is presented. The model takes into account the effects of the closed loop, namely average transmit power rise, power headroom, and variable soft hand-off gains, which are not considered in existing power control models. The power control problem is analyzed in this setting, and a number of properties are derived which generalize results from the classical model without closed-loop effects. Based on this analysis, an algorithm is given that determines required transmit powers taking into account these effects. This algorithm is a generalization of classical iterative algorithms without closed-loop effects. Finally, simulation results are presented to assess the validity of the approach.
\end{abstract}

Index Terms-Power control, code-division multiaccess, land mobile radio cellular systems.

\section{INTRODUCTION}

$\mathbf{P}$ OWER CONTROL is an essential part of direct-sequence code-division multiple access (DS-CDMA) cellular networks. In current systems it is generally implemented as a combination of closed-loop power control (CLPC) based on signal-to-interference ratio (SIR), and an outer loop (OL) that adjusts the reference SIR used by the CLPC.

The combined action of CLPC and multipath fading gives rise to the following effects:

- Average transmit power rise: The average transmit power necessary to obtain a given SIR increases compared to a system without CLPC, due to the statistical relationship between multipath fading and transmit power [1].

- Power headroom: The required SIR increases when the average transmit power approaches the maximum available power, because of the power values dictated by the closed loop being occasionally clipped. This can be adequately described by a necessary minimum power headroom [1].

- Soft hand-off (SHO) gain ${ }^{1}$ : The fast selection or combining of the received signals during soft hand-off alters the values of the required SIR, as well as those of the average transmit power rise and required power headroom [2].

Manuscript received February 15, 2005; revised September 30, 2005 and July 30, 2006; accepted January 19, 2007. The associate editor coordinating the review of this paper and approving it for publication was R. M. Buehrer.

The authors are with the Signals, Systems and Radiocommunications Department, Polytechnic University, 28040 Madrid, Spain (e-mail: \{lmendo, hernando\}@grc.ssr.upm.es).

Digital Object Identifier 10.1109/TWC.2007.05094.

${ }^{1}$ In this paper, SHO gain is the variation in a given parameter when the user is in SHO, due to fast frame selection or signal combining.
Since these effects are related to fast variations in instantaneous attenuation and transmit power, they will be referred to as short-term aspects in this paper.

This paper proposes a system-level model of a DS-CDMA cellular network with CLPC. The novelty of this model is the incorporation of short-term aspects. As argued in the following, current models of the power control problem do not accurately deal with these issues; however, they can have a great impact on network performance.

The analysis of DS-CDMA systems is usually divided into link level and system level. The link level analyzes the transmission between the mobile terminal and its serving base station(s), explicitly modelling the features (closed loop and multipath variations) that give rise to the previously defined short-term effects. The system level analyzes network behaviour on a larger time scale. It makes use of link-level results to characterize transmission. It should be noted that the mentioned "short-term" effects actually take place at a system level, although they are caused by short-term (linklevel) variations.

Existing system-level approaches to the power control problem are based on a linear equation system which describes the SIR requirements of the active users [3] [4] [5]. These models do not take into account the short-term aspects described above. As a consequence, application of these models to a cellular network with CLPC may lead to inaccuracies. To give an idea of the importance of this, it is noted that the link-level results reported in [1] [2] [6] indicate a range of $0-2 \mathrm{~dB}$ for the values of the average transmit power rise. Failing to take this effect into account underestimates transmission powers and interference levels, and gives too optimistic capacity values [7]. Likewise, the previously cited references report power headroom values in the range $0-8 \mathrm{~dB}$. This translates into an equal reduction of the average power that can be transmitted by the mobile, which may have a significant impact on coverage and capacity. Lastly, SHO gains in required SIR, power rise and power headroom vary in the range $0-4 \mathrm{~dB}[6]$, and, as will be seen in Section II, their actual values depend on interference levels; this dependence should be incorporated in the model. Thus, short-term effects need to be considered in order to obtain accurate system-level results. As an example, [6] claims that, for the system assumed therein, neglecting these effects gives too optimistic capacity estimations, with deviations of up to $15 \%$.

Several capacity studies have been published [7] [8] [9] that partially take into account short-term aspects. However, 
an accurate analysis of power control in this general setting has not been carried out yet. Specifically, [7] neglects SHO gains in required SIR and in transmit power rise, and does not take into account the effect of power headroom; besides, it assumes that all users are identical (same service class and multipath conditions). The analysis in [8] also assumes equal required SIRs for all users, does not model power limitations, and makes significant approximations. A general description of a simulation approach is given, but it does not specify how powers are calculated or how short-term effects are taken into account. The simulation model in [9], which is further described in [10, Sec. 3.2], incorporates all mentioned short-term effects and provides an algorithm for computing required powers, but no analysis is made of the solutions to the power control problem or the convergence of the proposed algorithm ${ }^{2}$.

The contribution of this paper is the following. A systemlevel model of power control is presented that incorporates the effect of short-term aspects. Only the uplink is considered, and the base-station assignment is regarded as fixed. As will be seen, the power control problem becomes nonlinear. This raises the issues of existence, uniqueness and properties of solutions, which are addressed in the paper. Based on this model, an iterative algorithm is proposed to compute the required transmission powers, and its convergence is established.

The rest of the paper is organized as follows. The power control model is presented in Section II, and results are given on the existence, uniqueness and properties of a solution. An algorithm to find that solution is proposed and analyzed in Section III. The validity of the proposed model and of the algorithm are assessed in Section IV by comparing with simulation results. Finally, conclusions are given in Section V.

\section{The Power Control Problem With SHORT-TERM EFFECTS}

In the classical, system-level model for the uplink power control, each user is assigned to one base station. The shortterm issues mentioned in Section I are not considered, and desired quality is identified with a target SIR. In these conditions, the power control problem is expressed as a linear equation system. In order to include the system-level effect of short-term aspects, a more general approach is presented in this section.

In the sequel, unless otherwise stated, when referring to attenuations, SIRs, and powers, their (system-level) average values with respect to fast variations will be meant. When needed, the terms "average" or "instantaneous" will be used to specify.

Let $M$ be the number of base stations and $K$ the number of active users in the uplink of a DS-CDMA system with singleuser detection and pseudorandom code sequences. Each user's service type may be different. Let $\boldsymbol{\alpha}$ be the $M \times K$ attenuation matrix, where $\alpha(m, k)$ is the attenuation (link gain) from user $k$ to base station $m, 0<\alpha(m, k)<1$; and let $\nu$ be the noise vector, where $\nu(m)>0$ is the background noise power at

\footnotetext{
${ }^{2}$ Note that, although the description of the simulator in [10] states that convergence stems from the results in [4], those results are not applicable because [4] assumes a power control model without short-term effects.
}

base station $m$. The transmit power of user $k$ is denoted as $P(k)$.

The logical structure of this section is as follows. Firstly, a characterization of the short-term effects is obtained. Towards this end, the concept of base-station assignment is defined in a suitable way to take into account SHO gains (Section II-A); a general definition of quality is given, as a function of the received SIRs which satisfies certain properties (Section II-B); the properties of the average transmit power rise are investigated (Section II-C); as well as those of the transmit power limitations (Section II-D). Secondly, a number of assumptions are made (Section II-E) related to the short-term effects. The obtained characterization, together with the assumptions, will be essential in the derivation of the remaining results in the paper. Thirdly, the power control equations are formulated (Section II-F) and their behaviour analyzed (Section II-G), based on the previous characterization and assumptions.

\section{A. Base-station assignment}

The assignment of a mobile user $k, \mathcal{A}(k)$, is defined as the set of base stations that are receiving the mobile. Note that, even if selection diversity is applied in $\mathrm{SHO}$, so that the mobile is instantaneously being served by only one base station, the fact that this base station can be selected from a given set yields a SHO gain, which justifies the definition. The global assignment $\mathcal{A}$ is the ordered set $[\mathcal{A}(1), \ldots, \mathcal{A}(K)]$. For each base station $m$, it is assumed that there exists some $k$ with $m \in \mathcal{A}(k)$ (otherwise that base station can be removed from the description).

\section{B. Quality and received SIRs}

It is assumed, for the time being, that transmit power is not limited at the mobile. In Section II-D the effect of transmit power limitations will be addressed.

Let $\Gamma(m, k)$ denote the received SIR of user $k$ at base station $m$. The following will be considered: ${ }^{3}$

(Q1) Quality for user $k$ is a nonnegative, measurable parameter $q(k)$. This parameter is possibly different for each user, depending on service type. The target quality for user $k$ is a certain value for the parameter $q(k)$.

(Q2) $q(k)$ is a $C^{1}$ function $\lambda_{k}$ of $\{\Gamma(m, k), m \in \mathcal{A}(k)\}$. In practice, all parameters used as quality measures satisfy this property. Note that $\lambda_{k}$ varies with the assignment, multipath propagation conditions and, if in SHO, diversity scheme (selection or combining) for user $k$. The system quality function $\boldsymbol{\lambda}$ is defined as a $K$-vector function whose $k$-th component gives $q(k)$.

(Q3) $\lambda_{k}$ is a monotone increasing function of each of its variables. This is a consequence of the fact that quality is degraded when the SIR at a serving base station decreases. In addition, $\lambda_{k}(0, \ldots, 0)=0$. This is a convenient normalization (shift in the origin for the $q(k)$ parameter) which entails no loss of generality.

For $|\mathcal{A}(k)|=1,{ }^{4}$ the description reduces to a certain SIR required at the serving base station (as in the classical model),

\footnotetext{
${ }^{3}$ Each property or assumption will be named with a letter and a number, where the letter indicates the concept or variable to which it applies.

${ }^{4}|S|$ represents the cardinal (number of elements) of a finite set $S$.
} 
whereas for $|\mathcal{A}(k)|>1$ the function $\lambda_{k}$ reflects the SHO gain phenomenon: there are different combinations of SIRs compatible with a given quality.

For each user $k$, it is assumed that the target quality is strictly positive, i.e. greater than the "quality" that is achieved with no transmit power (otherwise that user can be removed from the description).

\section{Average transmit power rise}

The transmit power rise phenomenon associated to CLPC consists in an increase in the average transmit power required for a given average received power (or SIR), caused by the correlation between instantaneous transmit power and instantaneous attenuation [1]. The transmit power rise may produce an increase in received interference, as is now discussed.

Let $B(k)$ denote the average transmit power rise of user $k$ due to CLPC. For $\mathcal{A}(k)=\{m\}$, the increased transmit power does not result in a higher received power at base station $m$, but in a more stable instantaneous SIR. Compared to a system without CLPC, the ratio of average received power at base station $m$ to average transmit power is thus divided by $B(k)$. This does not happen for other base stations, for which there is no correlation between instantaneous transmit power and instantaneous attenuation. According to this, received powers can be obtained as follows: for a given $P(k)$ (which includes the average transmit power rise), the average received power at base station $m$ is given by $\alpha(m, k) P(k) / B(k)$, whereas at base station $n \neq m$ it is $\alpha(n, k) P(k)$. The power rise $B(k)$ is thus sufficient to compute average received powers in this case. However, the situation becomes more complicated in SHO.

When the mobile is in SHO, each of the serving base stations compares the instantaneous received SIR against a reference value, sends "up" or "down" CLPC commands accordingly, and the mobile increases transmit power only if all received commands are "up". The effect is that, save for errors in the received commands, the mobile is instantaneously controlled by the base station which requires least power; and each base station has a certain probability of being instantaneously in control. As a result, the degree of correlation between instantaneous transmit power and instantaneous attenuation is not the same for all the serving base stations. If $m_{1}, m_{2} \in \mathcal{A}(k)$ with $\alpha\left(m_{1}, k\right)>\alpha\left(m_{2}, k\right)$ (average attenuations), most commands from base station $m_{1}$ will be "down", whereas most commands from $m_{2}$ will be "up"; thus the mobile will more often react to commands from $m_{1}$. Consider the event that the mobile $k$ is reacting to commands from a given base station $m \in \mathcal{A}(k)$. Conditioned on this event, the average value of the received power at $m$ shows no increase in spite of the transmit power rise. However, conditioned on the complementary event, the average received power at $m$ increases as much as the transmit power does. As a consequence, the unconditional average received power at base station $m$ will be $\alpha(m, k) P(k)$ multiplied by a certain coefficient, different for each serving base station, and not necessarily equal to $1 / B(k)$ (as it was in the case without SHO). These coefficients, as well as $B(k)$, depend in a complicate manner on the statistics of all the multipath channels between mobile $k$ and its serving base stations.
The described behaviour can be modelled by introducing an attenuation rise factor $\beta(m, k) \leq 1$, such that the power received from user $k$ at base station $m$ is $\alpha(m, k) \beta(m, k) P(k)$, with $\beta(m, k)=1$ if $m \notin \mathcal{A}(k)$, and $\beta(m, k)=1 / B(k)$ if $\mathcal{A}(k)=\{m\}$. The interference level $I(m, k)$ experienced by user $k$ at base station $m$ is

$$
I(m, k)=\sum_{\substack{j=1 \\ j \neq k}}^{K} \alpha(m, j) \beta(m, j) P(j)+\nu(m) .
$$

The properties of the attenuation rise factor are now analyzed. Consider a user $k$ with $\mathcal{A}(k)=\{m\}$. The instantaneous attenuation from user $k$ to base station $m$ has random, fast variations due to multipath. Interference, on the contrary, is approximately constant at a short-term level, because it aggregates all other signals and background noise. Assuming, for the moment, an ideal CLPC with a given reference SIR, the distribution of the instantaneous required transmit power, in a short-term observation interval (i.e. an interval of appreciable length compared to multipath variations, but very short on the system-level time scale), is determined by $I(m, k)$, the reference SIR and the statistics of the instantaneous attenuation. In particular, this distribution depends on transmit powers only through $I(m, k)$. Moreover, the shape of the distribution is independent of $I(m, k)$; increasing or decreasing the latter results only in a change of scale in the distribution.

For $|\mathcal{A}(k)|>1$, the attenuation rise factors are affected by SHO gains, as is now discussed. Within a given shortterm interval, each of the serving base stations has a certain probability of being effectively in control of the mobile, as discussed earlier. These probabilities depend in a complicate manner on the interference levels $I(n, k), n \in \mathcal{A}(k)$. However, if all these levels are increased in the same proportion, the distributions of the instantaneous powers required by the base stations are simply scaled in that proportion, and thus the referred probabilities do not change. On the other hand, if a certain $I(m, k)$ is decreased, with $I(n, k)$ maintained for all $n \neq m$, the probability associated to $m$ increases, which accentuates the attenuation rise between $m$ and $k$, i.e. reduces $\beta(m, k)$, whereas the contrary happens for the other serving base stations. Observe that, more generally, if the values $\alpha(n, k)$ are also regarded as variable, the preceding arguments apply with each $I(n, k)$ replaced by $I(n, k) / \alpha(n, k)$.

In a real system, the CLPC is affected by SIR measurement errors, power command demodulation errors, and loop delay. However, since these error sources are independent of power and interference levels, the behaviour claimed in the above paragraph is maintained.

As a result of the foregoing, the following properties hold for the attenuation rise factors:

(B1) For $\mathcal{A}(k)=\{m\}, \beta(m, k)$ does not depend on transmit powers.

(B2) For $|\mathcal{A}(k)|>1$, given $m \in \mathcal{A}(k), \beta(m, k)$ does not depend on $P(k)$, and depends on $P(j), j \neq k$ only through the interference levels $I(n, k), n \in \mathcal{A}(k)$. This dependence is such that:

- if $I(m, k)$ is increased while all other interference levels are maintained, $\beta(m, k)$ increases and $\beta(n, k)$ decreases for all $n \in \mathcal{A}(k), n \neq m$; 
- if the interference levels are changed in such a way that $\beta(m, k)$ increases, there exists $n \in \mathcal{A}(k)$ such that $\beta(n, k)$ decreases;

- if the interference levels $I(n, k)$ at all base stations $n \in \mathcal{A}(k)$ are increased in the same proportion, $\beta(m, k)$ does not change.

(B3) $\beta(m, k)=1$ for $m \notin \mathcal{A}(k)$.

Let $\boldsymbol{\beta}$ be the $M \times K$ attenuation rise matrix, whose components are the attenuation rise factors. From (B2), and making use of (1) and (B3), it stems that $\beta(m, k)$ is a function of $P(j)$ and $\beta(n, j), n \in \mathcal{A}(k), j \neq k$. This can be described by an attenuation rise function $\mathbf{b}$ such that $\boldsymbol{\beta}=\mathbf{b}(\mathbf{P}, \boldsymbol{\beta})$.

\section{Transmit power limitations}

Consider a maximum instantaneous transmit power $P_{\max }(k)$ for user $k$. If the average transmit power $P(k)$ is close to $P_{\max }(k)$, quality is degraded because of the instantaneous power being occasionally clipped at $P_{\max }(k)$; this requires increased SIRs in order to obtain the target quality.

This effect is studied in [1] and [6]. It is found that when the ratio $P_{\max }(k) / P(k)$, or "power headroom", is below a certain value, any further reduction in this ratio requires an equal [1] or even larger [6] increment $(\mathrm{dB})$ in received SIR to maintain a given quality. The amount of power rise, and thus attenuation rise, also exhibit dependence with power headroom, for approximately the same range of power headroom values. Finally, SHO gains in SIR and in attenuation rise are affected by low power headroom values as well.

These results indicate that, once $P(k)$ has reached a critical value, little or nothing can be done to increase quality, and it is reasonable to suspend any OL counteraction [1]. Such behaviour will be assumed in the sequel. This greatly simplifies the analysis. Namely, it can be considered that required SIRs and attenuation rise factors are independent of power headroom, provided that the latter is above a critical value; and below this value the target quality cannot be obtained, i.e. the power limitation has been reached. The critical value for the power headroom will be called power margin, $\mu(k)$. This gives an effective limit for $P(k)$ equal to $P_{\max }(k) / \mu(k) . \mu(k)$ is determined by the distribution function of instantaneous required power, and thus it shows the same type of dependence as the attenuation rise.

The above implies that the following properties hold:

(M1) For $|\mathcal{A}(k)|=1, \mu(k)$ does not depend on transmit powers.

(M2) For $|\mathcal{A}(k)|>1, \mu(k)$ does not depend on $P(k)$, and depends on $P(j), j \neq k$ only through the interference levels $I(n, k), n \in \mathcal{A}(k)$. This dependence is such that if these interference levels are increased in the same proportion, $\mu(k)$ does not change.

Let $\boldsymbol{\mu}$ denote the power margin vector, with components $\mu(k)$. As in Section II-C, a power-margin function $\mathbf{m}$ can be defined such that $\boldsymbol{\mu}=\mathbf{m}(\mathbf{P}, \boldsymbol{\beta})$.

\section{E. Assumptions}

The following assumptions are made in order to facilitate theoretical treatment. They are warranted in view of the behaviour of practical systems, as explained below.
(B4) $\beta(m, k)$ is a $C^{1}$ function of interference levels.

(B5) For $\mathcal{A}(k)=\left\{m_{1}, \ldots, m_{H}\right\}$ arbitrary, there exists $\theta<1$ such that if each $I\left(m_{h}, k\right), h=1, \ldots, H$ is multiplied by an arbitrary number $a_{h}>0, \beta(m, k)$ does not vary in a proportion outside the range $[1 /(\theta a), \theta a]$, with $a=$ $\max _{h}\left\{\max \left\{a_{h}, 1 / a_{h}\right\}\right\}$.

(B6) $\beta(m, k)$ has a nonzero lower bound.

(Q4) Suppose that $I(m, k)$ is increased, and all $I(n, k), n \in$ $\mathcal{A}(k), n \neq m$ are maintained. Then, in order to retain the desired quality, $\Gamma(n, k)$ must increase for all $n \in \mathcal{A}(k)$, $n \neq m$.

(M3) For $\mathcal{A}(k)=\left\{m_{1}, \ldots, m_{H}\right\}$, if each $I\left(m_{h}, k\right), h=$ $1, \ldots, H$ is multiplied by $a_{h}>0$, and this results in each $\beta\left(m_{h}, k\right)$ being multiplied by $b_{h}>0, \mu(k)$ does not vary in a proportion outside $[b / a, a / b]$, with $a=$ $\max _{h}\left\{\max \left\{a_{h}, 1 / a_{h}\right\}\right\}, b=\max _{h}\left\{\max \left\{b_{h}, 1 / b_{h}\right\}\right\}$.

Conditions (B4)-(B6) are mild, and can be expected to hold in practice.

As to condition (Q4), for $|\mathcal{A}(k)|=2$ it necessarily holds because of (Q3) and (B5). For $|\mathcal{A}(k)|>2$, it is justified as follows. If $I(m, k)$ is increased, (B2) and (B5) imply that $\beta(m, k)$ increases less than $I(m, k)$, and all other $\beta(n, k)$ decrease; besides, according to $(\mathrm{Q} 3)$, power must increase so that at least one of the SIRs $\Gamma(n, k)$ increases, with $\Gamma(m, k)$ decreased. What $(\mathrm{Q} 4)$ requires is that all $\Gamma(n, k), n \neq m$ increase, or equivalently that the SIR increases at the base station for which $\beta(n, k)$ most decreases. Since that base station is, from the viewpoint of attenuation rise, the one that most notices the increase in $I(m, k)$, it is plausible that its "contribution" to the user's quality is so essential that its SIR cannot decrease without degrading quality. In Section IV results will be given for a specific simulation model, and it will be seen that (Q4) is satisfied for $|\mathcal{A}(k)|=3$. The foregoing suggests that it presumably holds in practical systems.

In (M3), note that $b<a$ because of (B5). The assumption does not significantly reduce applicability.

\section{F. Power control equations}

The condition that each user $k$, with $\mathcal{A}(k)$ expressed as $\left\{m_{1}, \ldots, m_{H_{k}}\right\}$, obtains a target quality $q(k)$ is

$$
\begin{gathered}
\lambda_{k}\left(\Gamma\left(m_{1}, k\right), \ldots, \Gamma\left(m_{H_{k}}, k\right)\right)=q(k), \quad k=1, \ldots, K, \\
\Gamma(m, k)=\frac{\alpha(m, k) \beta(m, k) P(k)}{\sum_{j \neq k} \alpha(m, j) \beta(m, j) P(j)+\nu(m)} .
\end{gathered}
$$

In addition, the power constraints must be satisfied:

$$
P(k) \leq P_{\max }(k) / \mu(k), \quad k=1, \ldots, K .
$$

The functions $\lambda_{k}$ are nonlinear in general. Besides, attenuation rise factors and power margins depend on transmit powers (as discussed in Sections II-C and II-D). As a consequence, the equation system (2) is nonlinear.

It is noted that the computed SIRs $\Gamma(m, k)$ do not explicitly take into account the effect of "self-interference" caused by multipath in a Rake receiver. However, the degradation caused by multipath self-interference, and in general all non-ideal features of the receiver, are by definition included in the 
quality functions, i.e. $\lambda_{k}$ gives the actual quality taking into account such effects.

Let $\mathbf{P}$ be the transmit power vector, with components $P(k)$, and $\mathbf{q}$ the quality vector, defined analogously. The SIRs in (2) are functions of transmit powers and attenuation rise factors, which in turn depend on transmit powers. Therefore the equation system (2) can be expressed as

$$
\Lambda(\mathbf{P})=\mathbf{q}
$$

where the vector function $\Lambda$ explicitly describes all dependences. Since attenuation rise factors and qualities are $C^{1}$ functions of interference levels and SIRs respectively, $\boldsymbol{\Lambda}$ is a $C^{1}$ function.

\section{G. Properties}

The power control equations (2), or equivalently (3), establish a relationship between transmit powers and qualities. A quality vector $\mathbf{q}$ is feasible if there exists a solution ${ }^{5}$ $\mathbf{P}>0$; and $\mathbf{P}$ is then called a feasible solution. Proofs to all propositions to follow are given in the Appendix.

Proposition 1: Consider a cellular network with $M$ base stations and $K$ active users with attenuation matrix $\boldsymbol{\alpha}$, noise vector $\boldsymbol{\nu}$, in a given assignment $\mathcal{A}$ with quality function $\boldsymbol{\lambda}$, attenuation rise function $\mathbf{b}$, power-margin function $\mathbf{m}$, and with target quality vector $\mathbf{q}$.

1. If the equation system (2) has a feasible solution $\mathbf{P}$, it is the only feasible solution. Furthermore, this solution has a unique associated $\boldsymbol{\beta}$ matrix.

2. If $\mathbf{q}$ is feasible given a noise vector $\boldsymbol{\nu}_{1}>\mathbf{0}$, it is feasible for any noise vector $\boldsymbol{\nu}_{2}>\mathbf{0}$. Denoting by $\mathbf{P}_{1}$ and $\mathbf{P}_{2}$ the respective solutions, if $\boldsymbol{\nu}_{2} \leq \boldsymbol{\nu}_{1}$ with strict inequality in at least one component, then $\mathbf{P}_{2}<\mathbf{P}_{1}$.

3. Let $\mathbf{q}_{1}$ and $\mathbf{q}_{2}$ be quality vectors such that $\mathbf{q}_{2} \leq \mathbf{q}_{1}$ with strict inequality in at least one component. If (2) has a feasible solution $\mathbf{P}_{1}$ for the target quality vector $\mathbf{q}_{1}$, there is a feasible solution $\mathbf{P}_{2}$ for $\mathbf{q}_{2}$, and $\mathbf{P}_{2}<\mathbf{P}_{1}$.

These results generalize properties of the classical, linear model: feasible solutions are unique; feasibility is independent of noise; decreasing target qualities does not prevent feasibility, and reduces required powers.

Although noise powers are always positive in practice, it is now convenient to extend the model allowing for zero noise powers. A bar will denote the "extended" noise powers. Note, however, that the concept of feasibility requires positive noise powers.

Proposition 2: Given a cellular network with $M, K, \boldsymbol{\alpha}$, $\bar{\nu} \geq 0, \mathcal{A}, \boldsymbol{\lambda}, \mathbf{b}, \mathbf{m}$, and $\mathbf{q}$, for every $\mathbf{P}$ there is a unique compatible $\boldsymbol{\beta}$, i.e. there is a unique $\boldsymbol{\beta}$ such that $\boldsymbol{\beta}=\mathbf{b}(\mathbf{P}, \boldsymbol{\beta})$.

Based on this result, let $\mathcal{L}$ be defined as the set of quality vectors that can be obtained with zero noise powers,

$$
\left.\mathcal{L}=\left\{\mathbf{q} \in \mathbb{R}_{+}^{K} \mid \exists \mathbf{p} \in \mathbb{R}_{+}^{K} \text { with } \mathbf{q}=\boldsymbol{\Lambda}(\mathbf{p})\right\rfloor_{\bar{\nu}=\mathbf{0}}\right\} .
$$

Further, let $\mathcal{F}$ be the set of feasible quality vectors.

Proposition 3: Consider a cellular network with $M, K, \boldsymbol{\alpha}$, $\boldsymbol{\nu}>\mathbf{0}, \mathcal{A}, \boldsymbol{\lambda}, \mathbf{b}$, and $\mathbf{m}$.

\footnotetext{
${ }^{5}$ The convention is adopted that inequalities between vectors $\mathbf{x}, \mathbf{y}$ of the form $\mathbf{x}<\mathbf{y}, \mathbf{x} \leq \mathbf{y}$ or their inverses are interpreted componentwise.
}

1. For $\mathbf{q} \in \mathcal{F}$ and $\boldsymbol{\nu} \in \mathbb{R}_{+}^{K}$,

- $\mathbf{P}$ is a $C^{1}$ function of $\mathbf{q}$ with $\partial P(j) / \partial q(k)>0$;

- $\mathbf{P}$ is a $C^{1}$ function of $\boldsymbol{\nu}$ with $\partial P(j) / \partial \nu(m)>0$;

- $\operatorname{det}(\partial \boldsymbol{\Lambda} / \partial \mathbf{P})$ is a positive, continuous function of $q$ and $\nu$.

2. $\mathcal{L}$ satisfies the following properties:

- $\mathcal{L}$ is a simply connected $C^{0}$ manifold of dimension $K-1$.

- For any $\mathbf{q}_{0}>0$, there is a unique $a_{0}>0$ such that $a_{0} \mathbf{q}_{0} \in \mathcal{L}$.

- $\mathbf{q} \in \mathcal{L}$ if and only if $\operatorname{det}(\partial \boldsymbol{\Lambda} / \partial \mathbf{P})\rfloor_{\mathbf{q}}=0$.

3. $\mathcal{F}$ is simply connected, open, and extends from $\mathcal{L}$ inwards to the coordinate hyperplanes.

Again, this proposition generalizes results from the classical model: within $\mathcal{F}$, powers are $C^{1}$, increasing functions of target qualities and noise powers; $\mathcal{F}$ is open and simply connected; and it is limited by a hypersurface $\mathcal{L}$ formed by the quality vectors achieved with zero noise powers, or equivalently by the singular points of the mapping from powers to qualities. It can be verified that in the classical model $\mathcal{L}$ reduces to the hypersurface given in [11, Prop. 1].

\section{Algorithm FOR COMPUtATion OF TRANSMIT POWERS}

It has been shown in Section II that the power control equations have a unique feasible solution for target qualities within a certain region $\mathcal{F}$. An iterative algorithm to find this solution, taking into account power constraints, is now given. Let $\mathbf{P}_{\max }$ be a vector with components $P_{\max }(k)$.

Algorithm 1: Given $M, K, \boldsymbol{\alpha}, \boldsymbol{\nu}, \mathbf{P}_{\max }, \mathcal{A}, \boldsymbol{\lambda}, \mathbf{b}, \mathbf{m}$, and q, with $\mathcal{A}(k)$ expressed as $\left\{m_{1}, \ldots, m_{H_{k}}\right\}$ :

1. Choose initial values $\mathbf{P}^{0}$ and $\boldsymbol{\beta}^{0}$, and set $i=1$.

2. Update attenuation rise factors: $\boldsymbol{\beta}^{i}=\mathbf{b}\left(\mathbf{P}^{i-1}, \boldsymbol{\beta}^{i-1}\right)$.

3. Update power margins: $\boldsymbol{\mu}^{i}=\mathbf{m}\left(\mathbf{P}^{i-1}, \boldsymbol{\beta}^{i-1}\right)$.

4. Obtain the required powers $\hat{P}^{i}(k)$, with interference levels from the previous iteration:

$$
\begin{aligned}
& \lambda_{k}\left(\frac{\alpha\left(m_{1}, k\right) \beta^{i}\left(m_{1}, k\right) \hat{P}^{i}(k)}{\sum_{j \neq k} \alpha\left(m_{1}, j\right) \beta^{i-1}\left(m_{1}, j\right) P^{i-1}(j)+\nu\left(m_{1}\right)}, \ldots,\right. \\
& \left.\frac{\alpha\left(m_{H_{k}}, k\right) \beta^{i}\left(m_{H_{k}}, k\right) \hat{P}^{i}(k)}{\sum_{j \neq k} \alpha\left(m_{H_{k}}, j\right) \beta^{i-1}\left(m_{H_{k}}, j\right) P^{i-1}(j)+\nu\left(m_{H_{k}}\right)}\right) \\
& =q(k) .
\end{aligned}
$$

5. Update powers, taking into account power limitations: $P^{i}(k)=\min \left\{\hat{P}^{i}(k), P_{\max }(k) / \mu^{i}(k)\right\}$.

6. Increase $i$ and go back to step 2 , or finish if a given exit condition is satisfied.

Proposition 4: If $\mathbf{q}$ is feasible and the solution $\mathbf{P}$ is compatible with power restrictions, the algorithm converges to $\mathbf{P}$ from any $\mathbf{P}^{0}$ and $\boldsymbol{\beta}^{0}$. Otherwise, it converges to a unique point where, for each user $k$, either $q(k)$ is achieved or $P(k)$ equals the limit value $P_{\max }(k) / \mu(k)$.

The algorithm and the convergence result generalize those in [3], valid in the classical model. As in that case, each power is updated using interference levels from the previous iteration; however, in the general model with short-term effects, other parameters describing these effects are also updated. 
TABLE I

SIMULATION PARAMETERS

\begin{tabular}{|l|r|}
\hline Multipath channel model & 2 path, classical Doppler spectrum \\
\hline Receiver & Rake, ideal maximum-ratio combining \\
\hline CLPC period & $2 / 3 \mathrm{~ms}$ \\
CLPC step & Fixed, $1 \mathrm{~dB}$ \\
CLPC delay & 1 period \\
Error rate of CLPC commands & 0.01 \\
SIR estimation & Channel BER, 0.01 target \\
\hline Frame period & $20 \mathrm{~ms}$ \\
OL criterion & Gaussian error, mean 0, standard deviation $1 \mathrm{~dB}$ \\
OL period & $0.1 \mathrm{~dB}(1 \mathrm{~dB}$ down for power margin) \\
OL step & error probability in estimating a bit error \\
BER estimation & I: $12.2 \mathrm{~kb} / \mathrm{s} \mathrm{source} \mathrm{rate,} 60 \mathrm{~kb} / \mathrm{s}$ channel rate \\
\hline Service types & II: $64 \mathrm{~kb} / \mathrm{s}$ source rate, $240 \mathrm{~kb} / \mathrm{s}$ channel rate \\
\hline Maximum power & $21 \mathrm{dBm} \mathrm{for} \mathrm{service} \mathrm{I,} \mathrm{24} \mathrm{dBm} \mathrm{for} \mathrm{service} \mathrm{II}$ \\
\hline Spreading bandwidth & $5 \mathrm{MHz}$ \\
\hline Path loss & $144.4+38.4 d(\mathrm{~km})$ \\
Shadow fading standard deviation & $8 \mathrm{~dB}$ \\
Total gain (antenna, cable, terminal) & $10 \mathrm{~dB}$ \\
\hline Base-station assignment criterion & Minimum attenuation loss \\
SHO window & $6 \mathrm{~dB}$ \\
\hline Simulation area & {$[0,4] \times[0,4](\mathrm{km})$} \\
Base station location & $(i-0.5, j-0.5), i, j=1, \ldots, 4(\mathrm{~km})$ \\
User position, service and Doppler spread & Random, uniformly distributed \\
\hline
\end{tabular}

TABLE II

EFFECT OF POWER LIMITATION, $|\mathcal{A}(k)|=1$, SERVICE I, 3 KM/H.

\begin{tabular}{|r||c|c|c|c|c|c|c|}
\hline$P_{\max }(k) / P^{*}(k)(\mathrm{dB})$ & 0 & 2 & 4 & 6 & 8 & 10 & $\infty$ \\
\hline$P_{\max }(k) / P(k)(\mathrm{dB})$ & 5.9 & 5.9 & 6.2 & 6.9 & 8.2 & 9.8 & - \\
\hline Attenuation rise factor $(\mathrm{dB})$ & -2.2 & -2.2 & -2.2 & -2.4 & -2.5 & -2.7 & -2.8 \\
\hline
\end{tabular}

TABLE III

EFFECT OF POWER LIMITATION, $|\mathcal{A}(k)|=2$, SERVICE I, $3 \mathrm{KM} / \mathrm{H}$.

\begin{tabular}{|r||c|c|c|c|c|c|}
\hline$P_{\max }(k) / P^{*}(k)(\mathrm{dB})$ & 0 & 2 & 4 & 6 & 8 & $\infty$ \\
\hline$P_{\max }(k) / P(k)(\mathrm{dB})$ & 4.5 & 4.6 & 5.1 & 6.3 & 8.2 & - \\
\hline Attenuation rise factor $(\mathrm{dB})$ & -1.0 & -0.9 & -1.0 & -1.1 & -1.2 & -1.3 \\
\hline
\end{tabular}

The algorithm computes $\mathbf{P}$ using the known functions $\boldsymbol{\lambda}$, $\mathbf{b}$ and $\mathbf{m}$. In addition, it can be interpreted as a system-level description of the power-control interactions in an actual network, where those functions are performed by the CLPC and OL processes of all users (note that the processes for different users are coupled through interference levels, therefore their interactions are adequately described by means of short-term averages). From this viewpoint, Proposition 4 shows that the coupled power control processes converge in average powers.

\section{Simulation}

In this section, the validity of the algorithm is assessed, by comparing the transmit powers computed by the algorithm with those obtained from a simulation of the network. The simulation model is first described (Section IV-A). A link characterization for application of the algorithm is then derived (Section IV-B), and finally results are presented and discussed (Section IV-C).

\section{A. Simulation model}

The simulation considers a given set of active users, and models the power transmitted by each user at each CLPC interval. A simplified simulation model is used. Average attenuations are fixed, whereas their instantaneous values vary according to multipath fading. SHO is considered between two base stations only. The CLPC processes of all users are synchronous, which is a good approximation since propagation delays are much smaller than the CLPC period.

$\mathrm{OL}$ is based on channel bit-error-rate (BER). The reference SIR is adjusted in $0.1 \mathrm{~dB}$ steps. In addition, if the fraction of CLPC intervals with maximum transmit power reaches $10 \%$ within an OL interval, the reference SIR is decreased by 1 $\mathrm{dB} .{ }^{6}$ In SHO, the received frame with the minimum number of channel errors is selected; channel BER is estimated after selection; and a common reference SIR is sent to the serving base stations.

A network of 16 omnidirectional base stations is simulated, with two service classes. Three different Doppler spreads are considered, corresponding to 3,30 and $120 \mathrm{~km} / \mathrm{h}$ in the 2 $\mathrm{GHz}$ band. The simulated time is $300 \mathrm{~s}$, in order to accurately estimate averages. Other simulation parameters are shown in

\footnotetext{
${ }^{6}$ This is to approximately achieve the OL behaviour stated in Section II-D, and assumes that the mobile has a means to signal this "power too high" event to the network.
} 
TABLE IV

LINK CHARACTERIZATION FOR $|\mathcal{A}(k)|=2$, SERVICE I, 3 KM/H.

\begin{tabular}{|r||c|c|c|c|c|c|c|}
\hline$r_{1,2}(\mathrm{~dB})$ & 0 & 2 & 4 & 6 & 8 & 10 & $\infty$ \\
\hline Required SIR, base station 1 $(\mathrm{dB})$ & -22.7 & -22.1 & -21.8 & -21.5 & -21.4 & -21.4 & -21.3 \\
\hline Required SIR, base station 2 $(\mathrm{dB})$ & -22.7 & -23.6 & -24.6 & -25.9 & -27.4 & -29.0 & - \\
\hline Attenuation rise factor, 1 (dB) & -1.3 & -1.5 & -1.9 & -2.1 & -2.4 & -2.6 & -2.8 \\
\hline Attenuation rise factor, 2 (dB) & -1.3 & -0.9 & -0.7 & -0.4 & -0.3 & -0.3 & - \\
\hline Power margin (dB) & 4.6 & 4.6 & 4.8 & 5.1 & 5.4 & 5.7 & 5.9 \\
\hline
\end{tabular}

TABLE V

Link CHARACTERIZATION: Required SiRs FOR $|\mathcal{A}(k)|=3$, SERVICE I, 3 KM/H. AlL VALUes IN dB.

\begin{tabular}{|c||c|c|c|c|c|c|}
\hline$r_{1,2} r_{1,3}$ & 0 & 2 & 4 & 6 & 8 & 10 \\
\hline \hline 0 & $-23.4-23.4-23.4$ & $-23.0-23.0-24.6$ & $-22.8-22.8-26.0$ & $-22.8-22.8-27.7$ & $-22.7-22.7-29.5$ & $-22.7-22.7-31.4$ \\
\hline 2 & $-23.0-24.6-23.0$ & $-22.5-24.1-24.1$ & $-22.3-23.8-25.2$ & $-22.2-23.6-27.0$ & $-22.1-23.5-28.7$ & $-22.1-23.5-30.6$ \\
\hline 4 & $-22.8-26.0-22.8$ & $-22.3-25.2-23.8$ & $-22.0-24.9-24.9$ & $-21.9-24.6-26.4$ & $-21.7-24.6-28.0$ & $-21.8-24.5-30.0$ \\
\hline 6 & $-22.8-27.7-22.8$ & $-22.2-27.0-23.6$ & $-21.9-26.4-24.6$ & $-21.6-26.1-26.1$ & $-21.6-26.0-27.8$ & $-21.5-26.0-29.6$ \\
\hline 8 & $-22.7-29.5-22.7$ & $-22.1-28.7-23.5$ & $-21.7-28.0-24.6$ & $-21.6-27.8-26.0$ & $-21.5-27.5-27.5$ & $-21.4-27.7-29.2$ \\
\hline 10 & $-22.7-31.4-22.7$ & $-22.1-30.6-23.5$ & $-21.8-30.0-24.5$ & $-21.5-29.6-26.0$ & $-21.4-29.2-27.7$ & $-21.4-29.2-29.2$ \\
\hline
\end{tabular}

TABLE VI

COMPARISON BETWEEN ALGORITHM AND SIMULATION.

\begin{tabular}{|r||c|c|c|c|}
\hline$K$ & 100 & 160 & 200 & 220 \\
\hline Noise rise, average $(\mathrm{dB})$ & 1.9 & 3.5 & 5.1 & 7.1 \\
\hline Mean error $(\mathrm{dB})$ & 0.001 & 0.02 & 0.08 & 0.15 \\
\hline RMS error $(\mathrm{dB})$ & 0.10 & 0.12 & 0.17 & 0.25 \\
\hline
\end{tabular}

Table I. Note that, although the simulation model has been kept simple, the simplifications made do not jeopardise the comparison between simulation results and those obtained from the algorithm.

\section{B. Link characterization}

The link-level characterization is obtained by simulating a single user and observing required SIRs, attenuation rise factors and power margin (including their SHO gains). The attenuation rise factor is computed as the average received power divided by the product of average transmit power and average attenuation. Regarding the power margin, it is first noted that the simulated OL effectively limits average power at a fixed value. This is illustrated by Tables II and III, which show, in two specific situations, the resulting $P_{\max }(k) / P(k)$ as a function of $P_{\max }(k) / P^{*}(k)$, where $P^{*}(k)$ is the average power that would be obtained without limitations. It is seen that $P_{\max }(k) / P(k)$ equals $P_{\max }(k) / P^{*}(k)$ when the latter is high enough (no power clipping), whereas $P_{\max }(k) / P(k)$ is approximately constant when $P_{\max }(k) / P^{*}(k)$ is low, with a sharp transition between the two zones. This constant value is the power margin. In view of Tables II and III, $\mu(k)$ is computed as the value of $P_{\max }(k) / P(k)$ for $P_{\max }(k) / P^{*}(k)=2$ $\mathrm{dB}$.

For a SHO user $k$ assigned to base stations $m_{1}$ and $m_{2}$, from Section II-C it stems that SHO gains depend on the ratio $r_{1,2}=\alpha\left(m_{1}, k\right) I\left(m_{2}, k\right) /\left(\alpha\left(m_{2}, k\right) I\left(m_{1}, k\right)\right)$. Due to symmetry, it suffices to consider $r_{1,2}>0 \mathrm{~dB}$.

The obtained link-level results satisfy the assumptions in Section II-E. Table IV shows values for one of the Doppler spreads and one of the services; values for the other cases are omitted for brevity. Note that these link-level results define the functions $\boldsymbol{\lambda}, \mathbf{b}$ and $\mathbf{m}$.

Although not required for the intended comparison, link results have also been obtained for SHO between three base stations, in order to test (Q4) in this situation. It has been found that the assumption holds. As an illustration, this can be observed in Table $\mathrm{V}$, which shows results for the same conditions as in Table IV. For example, moving from left to right along any row corresponds to increasing the interference at base station 3 , while maintaining the interference at base stations 1 and 2; it is seen that this requires increased SIRs at base stations 1 and 2 (within the simulation accuracy).

\section{Results and discussion}

Series of 10 simulations are carried out, with a different value of $K$ in each series. The resulting average transmit powers are compared to those obtained using the algorithm with the link-level characterization. Results are given in Table VI, in terms of mean error and root-meansquare (RMS) error. The averaged noise rise [8] (given by $\left.\sum_{k=1}^{K} \alpha(m, k) \beta(m, k) P(k) / \nu(m)+1\right)$ is also shown for reference. It is seen that the considered values of $K$ cover the range of noise rise values usually found in practice. Very good agreement is observed between the algorithm and the simulation values, with low mean and RMS errors in all cases. The deviation between algorithm and simulation results is seen to slightly increase with system load. This is caused by the approximate modelling of the effect of transmit power limitations.

As indicated, all the assumptions on which the model is based hold in the considered simulation scenario. In addition, it can be seen that the link-level results in [1], [2] and [6] also satisfy these assumptions, which provides further support of their claimed mildness.

The proposed power control model incorporates short-term effects by means of the link-level characterization (functions $\lambda, \mathbf{b}$ and $\mathbf{m}$ ). The agreement between algorithm and simulation confirms the validity of this approach, corroborating that 
the model adequately captures the system-level effect of the short-term aspects associated to the closed loop.

\section{Conclusions}

The system-level power control problem has been formulated considering closed-loop effects, namely average transmit power rise, power headroom, and variable SHO gains. This approach generalizes existing models, and gives rise to a nonlinear equation system, whose properties have been analyzed. Under certain conditions, uniqueness of a feasible solution is assured, with properties similar to those of the classic, linear model. An iterative algorithm has been given that globally converges to that solution.

The proposed model and algorithm can be useful in network analysis and simulation, and in particular for radio planning tools. In addition, the algorithm can be interpreted as a systemlevel description of CLPC interactions, showing that in an actual network with CLPC the average powers converge.

\section{APPENDIX}

\section{ProOFS OF PROPOSITIONS}

Let the $M \times K$ matrix $\tau$ be defined as $\tau(m, k)=$ $\beta(m, k) P(k)$.

Lemma 1: Any $\boldsymbol{\tau}$ has unique associated $\mathbf{P}$ and $\boldsymbol{\beta}$.

Proof: Let $\mathbf{P}, \boldsymbol{\beta}, \mathbf{P}^{\prime}, \boldsymbol{\beta}^{\prime}$ be such that $\beta^{\prime}(n, j) P^{\prime}(j)=$ $\beta(n, j) P(j)$ for all $n, j$. Assume there are $m$ and $k$ with $\beta^{\prime}(m, k)>\beta(m, k)$. Then necessarily $m \in \mathcal{A}(k)$, and (B2) implies that there is $n \in \mathcal{A}(k)$ with $\beta^{\prime}(n, k)<\beta(n, k)$. Since $\beta^{\prime}(m, k) P^{\prime}(k)=\beta(m, k) P(k)$, we must have $P^{\prime}(k)<P(k)$, whereas $\beta^{\prime}(n, k) P^{\prime}(k)=\beta(n, k) P(k)$ requires $P^{\prime}(k)>$ $P(k)$, and a contradiction is obtained. Thus $\boldsymbol{\beta}^{\prime}=\boldsymbol{\beta}$, and then $\mathbf{P}^{\prime}=\mathbf{P}$.

Proof of Proposition 1.1: Let $\mathbf{P}_{0}$ be a solution of (2), with associated $\boldsymbol{\beta}_{0}$ and $\boldsymbol{\tau}_{0}$. Consider the mapping $\boldsymbol{\tau}^{i}=$ $\mathcal{I}\left(\boldsymbol{\tau}^{i-1}\right)$ such that $\tau^{i}(m, k)=\beta^{i}(m, k) \hat{P}^{i}(k)$, where $\beta^{i}(m, k)$ and $\hat{P}^{i}(k)$ are given as in steps 2 and 4 of the algorithm. $\tau_{0}$ is a fixed point of $\mathcal{I}$. From (Q3), (Q4) and (B2), $\mathcal{I}$ is standard [3]. Therefore $\boldsymbol{\tau}_{0}$ is unique, and from Lemma $1, \mathbf{P}_{0}$ and $\boldsymbol{\beta}_{0}$ are unique.

Lemma 2: If a system with target quality vector $\mathbf{q}_{0}$ and noise power vector $\boldsymbol{\nu}_{0}$ has a solution $\mathbf{P}_{0}$ with associated $\boldsymbol{\beta}_{0}$, then for any $a>0, a \mathbf{P}_{0}$ is a solution for the system with quality vector $\mathbf{q}_{0}$ and noise power vector $a \boldsymbol{\nu}_{0}$, and this solution has the same associated $\boldsymbol{\beta}_{0}$.

Proof: This is a straightforward consequence of (B2).

Proof of Proposition 1.2: Let $\boldsymbol{\nu}_{1}, \boldsymbol{\nu}_{2}>\mathbf{0}$, with $\nu_{1}(n) \neq$ $\nu_{2}(n)$ for some $n$, and let $\mathbf{P}_{1}$ be the solution for $\boldsymbol{\nu}_{1}$, with associated $\boldsymbol{\beta}_{1}$. We define $a=\max \left\{\max _{m}\left\{\nu_{2}(m) / \nu_{1}(m)\right\}, 1\right\}$ and $\boldsymbol{\nu}_{2}^{\prime}=a \boldsymbol{\nu}_{1} \geq \boldsymbol{\nu}_{1}$. From Lemma 2, $a \mathbf{P}_{1}$ is a solution for $\boldsymbol{\nu}_{2}^{\prime}$, and from part 1 of this proposition it is unique. If $\boldsymbol{\nu}_{2}^{\prime}=\boldsymbol{\nu}_{2}, \boldsymbol{\nu}_{2}$ is feasible. Assume that there is $m$ with $\nu_{2}(m)<\nu_{2}^{\prime}(m)$. Consider a mapping $\mathcal{I}_{P}$ that to each $\mathbf{P}^{i-1}$ and $\boldsymbol{\beta}^{i-1}$ assigns a matrix $\boldsymbol{\beta}^{i}$ and a power vector with components $\hat{P}^{i}(k)$ as in steps 2 and 4 of the algorithm. Replacing $\nu_{2}^{\prime}(m)$ by $\nu_{2}(m)$ and then applying $\mathcal{I}_{P}$ iteratively yields a componentwise decreasing positive sequence, which must converge [12, p. 38]. The resulting fixed point of $\mathcal{I}_{P}$ is a solution for the modified noise vector, and it is componentwise lower than $a \mathbf{P}_{1}$. Repeating for each $m$ with $\boldsymbol{\nu}_{2}(m)<\boldsymbol{\nu}_{2}^{\prime}(m)$, a feasible solution $\mathbf{P}_{2}<a \mathbf{P}_{1}$ results for $\boldsymbol{\nu}_{2}$. If $\boldsymbol{\nu}_{2} \leq \boldsymbol{\nu}_{1}$, then $a=1$, and thus $\mathbf{P}_{2}<\mathbf{P}_{1}$.

Lemma 3: A system with $M$ base stations and $K$ users is equivalent to another ("split") system with $\sum_{k=1}^{K}|\mathcal{A}(k)|$ base stations, in which each user is assigned to base stations different to those of all other users. The equivalence holds in the following sense: one system if feasible if and only if the other is, and if they are feasible, they have the same solution.

Proof: Each base station $m$, with $J_{m}$ assigned users, is "split" into $J_{m}$ base stations with the same parameters and functions as the original one. The power control equations for the split system are then the same as for the original one.

Proof of Proposition 1.3: Let $\mathbf{P}_{1}$ be the solution for $\mathbf{q}_{1}$ and a certain $\boldsymbol{\nu}_{1}$, with associated $\boldsymbol{\beta}_{1}$, and let $I_{1}(m, k)$ denote the resulting interference values. Consider the equivalent split system given by Lemma 3 (this allows to independently modify noise powers for each user and base station). Let $\nu(m, k)$ denote the noise power for user $k$ at the split base station that corresponds to the original base station $m$. Consider a given user $k$, with assignment $\mathcal{A}(k)=\left\{m_{1}, \ldots, m_{H}\right\}$ in the original system, and an arbitrary $c_{k}>1$. In the split system, if $\nu\left(m_{1}, k\right), \ldots \nu\left(m_{H}, k\right)$ are increased from $\nu\left(m_{1}\right), \ldots, \nu\left(m_{H}\right)$ to values $\nu^{\prime}\left(m_{1}, k\right), \ldots, \nu^{\prime}\left(m_{H}, k\right)$ such that

$$
\begin{aligned}
& \frac{I_{1}\left(m_{1}, k\right)-\nu_{1}\left(m_{1}\right)+\nu^{\prime}\left(m_{1}, k\right)}{I_{1}\left(m_{1}, k\right)}=\cdots \\
& =\frac{I_{1}\left(m_{H}, k\right)-\nu_{1}\left(m_{H}\right)+\nu^{\prime}\left(m_{H}, k\right)}{I_{1}\left(m_{H}, k\right)}=c_{k}
\end{aligned}
$$

while keeping transmit powers fixed, the attenuation rise factors are unchanged, according to (B2), whereas the SIRs of user $k$ at all its serving base stations are divided by $c_{k}$, which produces a decrease in quality, according to (Q3). Thus, any $q_{2}(k)<q_{1}(k)$ can be reached by adequately choosing $c_{k}$. After that, restoring the original noise powers while maintaining the quality $q_{2}(k)$ requires, according to Proposition 1.2, $\mathbf{P}_{2}<\mathbf{P}_{1}$. This is repeated for all users $k$ with $q_{2}(k)<q_{1}(k)$. The final $\mathbf{P}_{2}$ is a solution for $\mathbf{q}_{2}$ in the split system with the original noise powers, and thus it is also a solution in the original system.

Proof of Proposition 2: For $\mathbf{P}$ fixed, (B5) implies that $\mathbf{b}(\mathbf{P}, \boldsymbol{\beta})$ is a contractive function of $\boldsymbol{\beta}$. Applying the Banach fixed point theorem [13, p. 236] establishes the result.

Lemma 4: $\mathbf{P}$ is a continuous function of $\boldsymbol{\nu}$.

Proof: Consider the function $d$ that to each $\mathbf{x}, \mathbf{y} \in \mathbb{R}_{+}^{K}$ assigns

$$
d(\mathbf{x}, \mathbf{y})=\max _{k}\left\{\left|\ln \frac{x(k)}{y(k)}\right|\right\} .
$$

It is easily seen that $d$ is a metric. Let $\nu_{0}$ be a noise vector with solution $\mathbf{P}_{0}$. Let $\epsilon>0$, and consider the set $V$ of all $\mathbf{P}$ such that $d\left(\mathbf{P}, \mathbf{P}_{0}\right)<\epsilon$. We will prove that there exists $\delta>0$ such that for any $\boldsymbol{\nu}_{1}$ with $d\left(\boldsymbol{\nu}_{1}, \boldsymbol{\nu}_{0}\right)<\delta$, its corresponding $\mathbf{P}_{1} \in V$. Any $\mathbf{P} \in V$ satisfies $e^{-\epsilon} \mathbf{P}_{0}<\mathbf{P}<e^{\epsilon} \mathbf{P}_{0}$. From Lemma $2, e^{-\epsilon} \mathbf{P}_{0}$ and $e^{\epsilon} \mathbf{P}_{0}$ are respectively the solutions for $e^{-\epsilon} \boldsymbol{\nu}_{0}$ and $e^{\epsilon} \boldsymbol{\nu}_{0}$, and then Proposition 1.2 implies that if $\boldsymbol{\nu}_{1}$ satisfies $e^{-\epsilon} \boldsymbol{\nu}_{0}<\boldsymbol{\nu}_{1}<e^{\epsilon} \boldsymbol{\nu}_{0}$, the solution $\mathbf{P}_{1} \in V$. Therefore, taking $\delta=\epsilon$ suffices.

Lemma 5: Given $\mathbf{q}_{0}>0$, there exists $a_{0}$ such that for all positive $a \leq a_{0}, a \mathbf{q}_{0}$ is feasible and $\left.\operatorname{det}(\partial \boldsymbol{\Lambda} / \partial \mathbf{P})\right\rfloor_{a \mathbf{q}_{0}}>0$. 
Proof: It is easily seen that for $\mathbf{q}=\mathbf{0}$ and any $\boldsymbol{\nu}>0$ the system (3) is solved by $\mathbf{P}=0$, and for all $m, n \in\{1, \ldots, M\}$, $j, k \in\{1, \ldots, K\}$

$$
\begin{aligned}
& \frac{\partial \Gamma(m, k)}{\partial P(j)}=\frac{\alpha(m, k) \beta(m, k)}{\nu(m)} \delta_{j, k} \\
& \frac{\partial \Gamma(m, k)}{\partial \beta(n, l)}=0 .
\end{aligned}
$$

Thus, from (Q3) it stems that the matrix $\partial \boldsymbol{\Lambda} / \partial \mathbf{P}$ has positive elements along its main diagonal and the rest are zero, and therefore its determinant is positive. The same holds for the inverse matrix. The inverse function theorem [12, p. 140] implies that (3) defines $\mathbf{P}$ as a $C^{1}$ function of $\mathbf{q}$ in a neighbourhood of $\mathbf{q}=\mathbf{0}, \mathbf{P}=\mathbf{0}$, with $\partial P(j) / \partial q(j)\rfloor_{\mathbf{q}=\mathbf{0}} \triangleq$ $d_{j}>0$ and $\left.\partial P(j) / \partial q(k)\right\rfloor_{\mathbf{q}=\mathbf{0}}=0$ for $j \neq k$. The continuity and positivity of $\partial P(j) / \partial q(j)$ at $\mathbf{q}=\mathbf{0}$ assure that there is a neighbourhood $U$ of $\mathbf{q}=\mathbf{0}$ where these derivatives are positive, and thus all points within $U$ have positive powers. Since $\operatorname{det}(\partial \boldsymbol{\Lambda} / \partial \mathbf{P})$ is a sum of products of the elements in $\partial \boldsymbol{\Lambda} / \partial \mathbf{P}$, which are also continuous because of (Q2), a similar argument shows that there is a neighbourhood $U^{\prime}$ of $\mathbf{q}=\mathbf{0}$ where the determinant is positive. The claimed result follows taking $a$ small enough that $a \mathbf{q}_{0}$ lies in $U \cap U^{\prime}$.

Lemma 6: $\mathbf{q}_{0} \in \mathcal{L}$ if and only if $\left.\operatorname{det}(\partial \boldsymbol{\Lambda} / \partial \mathbf{P})\right\rfloor_{\mathbf{q}_{0}}=0$.

Proof: "If". Let $\mathbf{q}_{0}>0$ and $\overline{\boldsymbol{\nu}}_{0} \geq 0$ such that $\operatorname{det}(\partial \boldsymbol{\Lambda} / \partial \mathbf{P})\rfloor_{\mathbf{q}_{0}, \overline{\boldsymbol{\nu}}_{0}}=0$. We will first show that $\mathbf{q}_{0}$ cannot be feasible. We proceed by contradiction. Suppose there are $\mathbf{P}_{0}>0$ and $\boldsymbol{\nu}_{0}>0$ such that $\left.\boldsymbol{\Lambda}\left(\mathbf{P}_{0}\right)\right\rfloor_{\boldsymbol{\nu}_{0}}=\mathbf{q}_{0}$. From Lemma 5, let $a_{0}>0$ such that $a_{0} \mathbf{q}_{0}$ is feasible with $\operatorname{det}(\partial \boldsymbol{\Lambda} / \partial \mathbf{P})\rfloor_{a_{0} \mathbf{q}_{0}, \boldsymbol{\nu}_{0}}>0$.

All points $a \mathbf{q}_{0}, a<1$ are feasible, according to Proposition 1.3, and thus $\operatorname{det}(\partial \boldsymbol{\Lambda} / \partial \mathbf{P})$ is well-defined at those points. We will prove that $\operatorname{det}(\partial \boldsymbol{\Lambda} / \partial \mathbf{P})\rfloor_{a \mathbf{q}_{0}, \boldsymbol{\nu}_{0}}$ is a continuous function of $a$ for $a_{0} \leq a \leq 1$. Consider $a$ in that interval, and let $\mathbf{P}$ be the solution for $a \mathbf{q}_{0}$. For any $b<1$ sufficiently close to $a, b \mathbf{q}_{0}$ can be achieved with powers $\mathbf{P}$, by considering the equivalent split system from Lemma 3 and adequately modifying its noise powers while keeping attenuation rise factors unchanged, as in the proof of Proposition 1.3. The modifications required in the noise powers are continuous functions of $b$. Restoring the original noise vector while maintaining the qualities $b \mathbf{q}_{0}$ requires a power vector which is a continuous function of the modifications that have been applied to the noise powers, according to Lemma 4. Combining both facts, the power vector $\mathbf{P}$ required for $a \mathbf{q}_{0}$ is seen to be a continuous function of $a$.

Since $\mathbf{P}$ is a continuous function of $a$ and $\boldsymbol{\Lambda}$ is a $C^{1}$ function of $\mathbf{P}, \operatorname{det}(\partial \boldsymbol{\Lambda} / \partial \mathbf{P})\rfloor_{a \mathbf{q}_{0}, \boldsymbol{\nu}_{0}}$ is a continuous function of $a$ in the interval $a_{0} \leq a \leq 1$. Thus, the set of values of $a$ within this interval for which the determinant is zero has a minimum, which will be denoted $a_{1}$. This implies that $\operatorname{det}(\partial \boldsymbol{\Lambda} / \partial \mathbf{P})\rfloor_{a \mathbf{q}_{0}, \boldsymbol{\nu}_{0}}>0$ for $a \in\left[a_{0}, a_{1}\right)$ and $\operatorname{det}(\partial \boldsymbol{\Lambda} / \partial \mathbf{P})\rfloor_{a \mathbf{q}_{0}, \boldsymbol{\nu}_{0}} \rightarrow 0$ as $a \rightarrow a_{1}{ }^{-}$. The inverse function theorem can be applied for all $a \in\left[a_{0}, a_{1}\right)$, with the result that $\operatorname{det}(\partial \mathbf{P} / \partial \mathbf{q})\rfloor_{a \mathbf{q}_{0}, \nu_{0}} \rightarrow \infty$ as $a \rightarrow a_{1}{ }^{-}$. This implies that there are $j, k$ such that $\partial P(k) / \partial q(j) \rightarrow \infty$. But this is impossible because $P(k)$ is continuous at $a_{1} \mathbf{q}_{0}$. Therefore the assumption that $\mathbf{q}_{0}$ is feasible is false.
We will now prove that $\overline{\boldsymbol{\nu}}_{0}=\mathbf{0}$. To this end, assume that $\bar{\nu}_{0}(m)>0$ for some $m$, and let $k$ be a user with $m \in \mathcal{A}(k)$. Consider the equivalent split system, with noise powers denoted as $\nu(n, j)$. In this system, the following modification is applied. First $\nu(m, k)$ is divided by an arbitrary $c>1$, without modifying transmission powers. This reduces the interference $I(m, k)$ seen at base station $m$ for user $k$, which in turn produces, according to (B2), a decrease in the attenuation rise factor $\beta(m, k)$ and an increase in $\beta(n, k)$ for all $n \in \mathcal{A}(k), n \neq m$. From (B5), the reduction in $\beta(m, k)$ is smaller than the reduction in $I(m, k)$. Therefore user $k$ has increased SIRs at all its serving base stations, and from (Q3) its quality is higher than required. Secondly, for all base stations $n \in \mathcal{A}(k)$ with $\nu(n, k)=0$ (if there is any), an arbitrarily small amount of noise is introduced; and $P(k)$ is lowered so that the quality obtained by user $k$ equals its target value. As a final step, the noise powers for other users are increased to compensate for the smaller $P(k)$, so that their interference levels and attenuation rise factors do not change. At the end of this process all noise powers in the split system are positive, and the desired quality $\mathbf{q}_{0}$ is achieved. This implies that $\mathbf{q}_{0}$ is feasible in the split system, and thus in the original system, in contradiction with our previous result. Therefore $\bar{\nu}_{0}(m)=0$ for all $m$.

"Only if". Given $\mathbf{q}_{0} \in \mathcal{L}$, let $\mathbf{P}_{0}>0$ with $\left.\boldsymbol{\Lambda}\left(\mathbf{P}_{0}\right)\right\rfloor_{\bar{\nu}=\mathbf{0}}=$ $\mathbf{q}_{0}$. From (B2), $\left.\boldsymbol{\Lambda}\left(a \mathbf{P}_{0}\right)\right\rfloor_{\bar{\nu}=\mathbf{0}}=\mathbf{q}_{0}$ for any $a>0$. This implies that $\partial \boldsymbol{\Lambda} / \partial \mathbf{P} \cdot \mathbf{P}_{0}=\mathbf{0}$, and therefore $\operatorname{det}(\partial \boldsymbol{\Lambda} / \partial \mathbf{P})=0$.

Lemma 7: For $\mathbf{q}_{0}$ feasible, $\operatorname{det}(\partial \boldsymbol{\Lambda} / \partial \mathbf{P})$ is a positive, continuous function of $\mathbf{q}_{0}$.

Proof: Let $\mathbf{q}_{0}$ be feasible. By Lemma 5, there exists $a_{0}<1$ such that $a_{0} \mathbf{q}_{0}$ is feasible with positive $\operatorname{det}(\partial \boldsymbol{\Lambda} / \partial \mathbf{P})$. From Proposition 1.3, all points $a \mathbf{q}_{0}, a \leq 1$ are feasible; from Lemma 6 they have nonzero determinant; and the inverse function theorem then implies that the determinant is continuous at these points. Therefore it must be positive at all points $a \mathbf{q}_{0}$, $a \leq 1$, and in particular at $\mathbf{q}_{0}$.

Proof of Proposition 3.1: From Lemma $7, \operatorname{det}(\partial \boldsymbol{\Lambda} / \partial \mathbf{P})$ is positive and continuous in $\mathcal{F}$. The inverse function theorem then assures that $\mathbf{P}$ is a $C^{1}$ function of $\mathbf{q}$. From the implicit function theorem [12, p. 148], $\mathbf{P}$ is a $C^{1}$ function of $\boldsymbol{\nu}>0$. Its partial derivatives are positive because of Proposition 1.2 and 1.3.

Lemma 8: Given any $\mathbf{q}_{0}>0$, there exists a unique number $a_{0}>0$ such that $a_{0} \mathbf{q}_{0} \in \mathcal{L}$, and $a_{0}$ is a continuous function of $\mathbf{q}_{0}$. A target $a \mathbf{q}_{0}$ is feasible if and only if $a<a_{0}$.

Proof: Let $\mathbf{q}_{0}>0$. From (B6), let $\beta_{\mathrm{lb}}>0$ be a lower bound for the attenuation rise factors of all users. Consider a matrix $\boldsymbol{\beta}^{i}$ with $\boldsymbol{\beta}^{i}(m, k) \in\left[\beta_{\mathrm{lb}}, 1\right]$ arbitrary for all $m, k$. For the moment, we will treat this matrix as if it were the actual attenuation rise matrix.

For any $k$, with $\mathcal{A}(k)=\left\{m_{k, 1}, \ldots, m_{k, H_{k}}\right\}$, the values $\beta^{i}\left(m_{k, 1}, k\right), \ldots, \beta^{i}\left(m_{k, H_{k}}, k\right)$ determine the interference levels $I\left(m_{1}, k\right), \ldots, I\left(m_{k, H_{k}}, k\right)$ up to a scaling factor, as is now proved. Assume there are $I\left(m_{k, 1}, k\right), \ldots, I\left(m_{k, H_{k}}, k\right)$ and $I^{\prime}\left(m_{k, 1}, k\right), \ldots, I^{\prime}\left(m_{k, H_{k}}, k\right)$ compatible with $\boldsymbol{\beta}^{i}$. Let $s_{h}=$ $I^{\prime}\left(m_{k, h}, k\right) / I\left(m_{k, h}, k\right)$ for $h=1, \ldots, H_{k}, s=\min _{h}\left\{s_{h}\right\}$ and $j=\arg \min _{h}\left\{s_{h}\right\}$. If $s_{h} \neq s$ for some $h$, (B2) implies that $\beta\left(m_{k, j}, k\right)$ is lower with the primed values, which is impossible. Therefore $s_{1}=s_{2}=\cdots=s_{H_{k}}$. 
As a consequence of the previous paragraph, $\boldsymbol{\beta}^{i}$ determines the proportion between the SIRs $\Gamma\left(m_{k, 1}, k\right), \ldots, \Gamma\left(m_{k, H_{k}}, k\right)$. With this fixed proportion, any target $q(k)$ is equivalent to a certain value for $\Gamma\left(m_{k, 1}, k\right)$. Furthermore, since the attenuation rise matrix is fixed, it can be simply "absorbed" into the attenuation matrix, which reduces the problem to that in the classical model. For an arbitrary $\gamma \in \mathbb{R}_{+}^{K}$ and zero noise powers, let us consider the problem of finding the maximum $c$, and a corresponding power vector, such that $\Gamma\left(m_{k, 1}, k\right)=c \gamma(k)$ for all $k$. If $\gamma(1)=\cdots=\gamma(K)$, it is shown in [14] that the maximum $c$ is $1 /(\chi-1)$, where $\chi>1$ is the dominant, simple eigenvalue of a certain matrix whose elements are attenuation ratios, and which can be assumed to be nonsingular with probability one. This eigenvalue has a positive eigenvector which is the power vector solution, determined up to a scaling factor. The case for arbitrary $\gamma$ can be reduced to this by splitting the base stations according to Lemma 3 and then "absorbing" $\gamma(1), \ldots, \gamma(K)$ into attenuations. Thus, for every $\gamma$ there is maximum $c$, denoted $c_{*}(\gamma)$. Since eigenvalues are continuous functions of the matrix elements, $c_{*}(\gamma)$ is a continuous function of $\gamma$. Furthermore, the corresponding eigenvector determined by setting its $K$-th entry to 1 is also a continuous function of $\gamma$.

Consider quality vectors of the form $d \mathbf{q}_{0}, d>0$. With zero noise powers and assuming a fixed attenuation rise matrix, increasing $d$ without bound requires arbitrarily high values of $\Gamma\left(m_{k, 1}, k\right), k=1, \ldots, K$ (not necessarily in a fixed proportion for different users). Thus, as $d$ is increased, a value $d_{*}$ is reached that corresponds to the $c_{*}\left(\gamma_{*}\right)$ for a certain $\gamma_{*}$. Let $\mathbf{P}^{i+1}$ be the corresponding power vector with $\mathbf{P}^{i+1}(K)=1$. (At this point the quality $d_{*} \mathbf{q}_{0}$ would be "achieved", only that $\boldsymbol{\beta}^{i}$ need not be the attenuation matrix associated to $\mathbf{P}^{i+1}$. This is solved in the following.) Let the mapping $\boldsymbol{\beta}^{i+1}=\mathcal{B}\left(\boldsymbol{\beta}^{i}\right)$ be defined as $\mathcal{B}\left(\boldsymbol{\beta}^{i}\right)=\mathbf{b}\left(\mathbf{P}^{i+1}, \boldsymbol{\beta}^{i}\right)$. Since $\mathbf{P}^{i+1}$ varies continuously with $\gamma_{*}$, which in turn varies continuously with $\boldsymbol{\beta}^{i}, \mathcal{B}$ is continuous. From (B6), attenuation rise matrices are defined in a compact, convex subset of the space of $M \times K$ real matrices, and thus the Brouwer fixed point theorem [13, p. 409] implies that $\mathcal{B}$ has a fixed point. The $d_{*}$ value for that particular attenuation rise matrix gives a quality vector $d_{*} \mathbf{q}_{0}$ which is achieved with zero noise powers, i.e. that $d_{*}$ is the desired $a_{0}$.

Let $\mathbf{P}_{0}$ be a power vector such that $\left.a_{0} \mathbf{q}_{0}=\boldsymbol{\Lambda}\left(\mathbf{P}_{0}\right)\right\rfloor_{\bar{\nu}=\mathbf{0}}$. From Lemma $6, \operatorname{det}(\partial \boldsymbol{\Lambda} / \partial \mathbf{P})\rfloor_{a_{0} \mathbf{q}_{0}}=\mathbf{0}$. Lemma 7 then implies that $a_{0} \mathbf{q}_{0}$ is not feasible, and from Proposition 3 neither is $a \mathbf{q}_{0}$ with $a>a_{0}$. For $a<a_{0}, a \mathbf{q}_{0}$ is feasible, because, considering the split system given by Lemma 3, and adequately modifying noise powers as in the proof of Proposition 1.3, $a \mathbf{q}_{0}$ can be achieved with $\mathbf{P}_{0}$ and positive noise powers. Thus $a_{0}$ is unique, and $a \mathbf{q}_{0}$ is feasible if and only if $a<a_{0}$.

We now prove that $a_{0}$ is a continuous function of $\mathbf{q}_{0}$. Given $\mathbf{q}_{0}$ and its corresponding $a_{0}$, let $\epsilon>0$ and $V=\left(a_{0}-\epsilon, a_{0}+\epsilon\right)$. Consider an arbitrary $\mathbf{q}_{1}$ and its corresponding $a_{1}$. Choosing $\mathbf{q}_{1}$ sufficiently close to $\mathbf{q}_{0}$, we can find $a_{1}^{\prime}, a_{1}^{\prime \prime} \in V$ such that $a_{1}^{\prime} \mathbf{q}_{1}<a_{0} \mathbf{q}_{0}$ and $a_{1}^{\prime \prime} \mathbf{q}_{1}>a_{0} \mathbf{q}_{0}$. From Proposition 1.3, $a_{1}^{\prime} \mathbf{q}_{1}$ is feasible and $a_{1}^{\prime \prime} \mathbf{q}_{1}$ is not. Our previous result then implies that $a_{1}^{\prime}<a_{1} \leq a_{1}^{\prime \prime}$, and thus $a_{1} \in V$.
Proof of Proposition 3.2: With Lemmas 6 and 8, it only remains to prove that $\mathcal{L}$ is a simply connected $C^{0}$ manifold of dimension $K-1$. Let $\zeta \in \mathbb{R}_{+}^{K-1}$, and consider $\mathbf{q}^{*}$ with $q^{*}(k)=\zeta(k)$ for $k=1, \ldots, K-1$ and $q^{*}(K)=1$. By Lemma 8 , there is $a^{*}$ with $a^{*} \mathbf{q}^{*} \in \mathcal{L}$, and $a^{*}$ is a continuous function of $\zeta$. Thus the function that to $\zeta$ assigns $a^{*} \mathbf{q}^{*}$ is a homeomorphism from $\mathbb{R}_{+}^{K-1}$ to $\mathcal{L}$. This establishes the desired result.

Proof of Proposition 3.3: It follows from Lemma 8.

Proof of Proposition 4: As in the proof of Lemma 4, consider the metric $d$ that to each pair of positive matrices $\tau_{1}, \tau_{2}$ assigns the value

$$
d\left(\boldsymbol{\tau}_{1}, \boldsymbol{\tau}_{2}\right)=\max _{m, k}\left\{\left|\ln \frac{\tau_{1}(m, k)}{\tau_{2}(m, k)}\right|\right\} .
$$

The space of $\tau$ matrices is complete with respect to $d$, and thus the Banach fixed point theorem can be applied. We will use it to show that, considering the iteration given by steps $2-5$ of the algorithm as a mapping in $\tau$, it converges to a unique fixed point.

Let us consider $\mathbf{P}_{1}, \boldsymbol{\beta}_{1}, \mathbf{P}_{2}$ and $\boldsymbol{\beta}_{2}$ arbitrary, and the corresponding $\boldsymbol{\tau}_{1}$ and $\boldsymbol{\tau}_{2}$. Let $\boldsymbol{\beta}_{1}^{\prime}, \boldsymbol{\mu}_{1}^{\prime}, \hat{\mathbf{P}}_{1}^{\prime}$ and $\mathbf{P}_{1}^{\prime}$ be obtained through one iteration on $\mathbf{P}_{1}$ and $\boldsymbol{\beta}_{1}$, and let $\hat{\boldsymbol{\tau}}_{1}^{\prime}$ and $\boldsymbol{\tau}_{1}^{\prime}$ correspond to $\hat{\mathbf{P}}_{1}^{\prime}, \boldsymbol{\beta}_{1}$ and $\mathbf{P}_{1}^{\prime}, \boldsymbol{\beta}_{1}$ respectively. $\boldsymbol{\beta}_{2}^{\prime}, \boldsymbol{\mu}_{2}^{\prime}, \hat{\mathbf{P}}_{2}^{\prime}$, $\mathbf{P}_{2}^{\prime}, \hat{\tau}_{2}^{\prime}$ and $\tau_{2}^{\prime}$ are analogously defined. We need to show that there is $\kappa<1$ such that $d\left(\boldsymbol{\tau}_{1}^{\prime}, \boldsymbol{\tau}_{2}^{\prime}\right) \leq \kappa d\left(\boldsymbol{\tau}_{1}, \boldsymbol{\tau}_{2}\right)$, for $i \geq 1$, with $\kappa$ independent of $\boldsymbol{\tau}_{1}, \boldsymbol{\tau}_{2}$. It suffices to consider $\boldsymbol{\tau}_{1} \neq \boldsymbol{\tau}_{2}$.

For any $n$, we define $I_{1}^{*}(n, k)=\sum_{j \neq k} \alpha(n, j) \tau_{1}(n, j)$, $I_{1}(n, k)=I_{1}^{*}(n, k)+\nu(n)$, and analogously $I_{2}^{*}(n, k)$, $I_{2}(n, k)$. Let $a=e^{d\left(\boldsymbol{\tau}_{1}, \boldsymbol{\tau}_{2}\right)}$ and $\nu_{\min }=\min _{n}\{\nu(n)\}$. As a consequence of $(\mathrm{Q} 3)$, given $k$, there is a minimum SIR $\Gamma_{\min }(k)$ that must be experienced in at least one $m \in \mathcal{A}(k)$ in order to achieve $q(k)$. Thus, defining $\Gamma_{\min }=\min _{k}\left\{\Gamma_{\min }(k)\right\}$, $\alpha_{\max }=\max _{m, k}\{\alpha(m, k)\}$ and $P_{\max }=\max _{k}\left\{P_{\max }(k)\right\}$, we have $P_{1}(k), P_{2}(k) \geq \nu_{\min } \Gamma_{\min } / \alpha_{\max } \triangleq P_{\min }$ for $i \geq 1$, and $a \leq P_{\max } /\left(\beta_{\mathrm{lb}} P_{\text {min }}\right) \triangleq a_{\text {max }}$.

Defining

$$
\begin{aligned}
I_{\max }^{*} & =\max _{n, k}\left\{\sum_{j \neq k} \alpha(n, j) P_{\max }(j)\right\} \\
c & =\frac{a I_{\max }^{*}+\nu_{\min }}{I_{\max }^{*}+\nu_{\min }}
\end{aligned}
$$

it is easily seen that, for any $n$ and $k, 1 / c \leq$ $I_{2}(n, k) / I_{1}(n, k) \leq c$. From (Q3), given $m$ and $k$ with $m \in \mathcal{A}(k), \hat{\tau}_{2}^{\prime}(m, k) / \hat{\tau}_{1}^{\prime}(m, k)$ is maximum when $I_{2}(n, k) / I_{1}(n, k)=c$ for all $n \in \mathcal{A}(k)$, and (B2) then implies that $\hat{\tau}_{2}^{\prime}(m, k)=c \hat{\tau}_{1}^{\prime}(m, k)$. For $m$ and $k$ with $m \notin \mathcal{A}(k)$, the arguments used in the proof of Proposition 1.2 show that $\hat{\tau}_{2}^{\prime}(m, k) / \hat{\tau}_{1}^{\prime}(m, k)=\hat{P}_{2}^{\prime}(k) / \hat{P}_{1}^{\prime}(k)$ is also bounded by $c$. Thus, for any $m, k$

$$
\frac{\hat{\tau}_{2}^{\prime}(m, k)}{\hat{\tau}_{1}^{\prime}(m, k)}=\frac{\beta_{2}^{\prime}(m, k) \hat{P}_{2}^{\prime}(m, k)}{\beta_{1}^{\prime}(m, k) \hat{P}_{1}^{\prime}(m, k)} \leq c .
$$

If $\hat{P}_{1}^{\prime}(k) \leq P_{\max }(k) / \mu_{1}^{\prime}(k)$, then $P_{1}^{\prime}(k)=\hat{P}_{1}^{\prime}(k)$, and (6) implies that

$$
\frac{\tau_{2}^{\prime}(m, k)}{\tau_{1}^{\prime}(m, k)} \leq c
$$

Else, (M3) implies (7). Therefore, this inequality is satisfied in all cases. 
Interchanging subindices in the above arguments leads to

$$
\frac{\tau_{2}^{\prime}(m, k)}{\tau_{1}^{\prime}(m, k)} \geq \frac{1}{c}
$$

Combining (7) and (8) and substituting $c$,

$$
\frac{\left|\ln \frac{\tau_{2}^{\prime}(m, k)}{\tau_{1}^{\prime}(m, k)}\right|}{\ln a} \leq \frac{\ln \frac{a I_{\max }^{*}+\nu_{\min }}{I_{\max }^{*}+\nu_{\min }}}{\ln a} \leq \frac{\ln \frac{a_{\max } I_{\max }^{*}+\nu_{\min }}{I_{\max }^{*}+\nu_{\min }}}{\ln a_{\max }} \triangleq b<1 .
$$

Taking $\kappa=(b+1) / 2<1$ (or any other value between $b$ and 1) establishes that the mapping is contractive. Thus the Banach fixed point theorem assures that it has a unique fixed point $\boldsymbol{\tau}$, for which Lemma 1 implies that $\mathbf{P}$ and $\boldsymbol{\beta}$ are also unique. From the definition of the algorithm, the obtained $\mathbf{P}$ has the properties stated in the proposition.

\section{ACKNOWLEDGMENTS}

The authors are grateful to the anonymous reviewers and to the editor, Dr. R. M. Buehrer, for their valuable comments.

\section{REFERENCES}

[1] K. Sipilä, J. Laiho-Steffens, A. Wacker, and M. Jäsberg, "Modeling the impact of the fast power control on the WCDMA uplink," in Proc. IEEE Vehicular Technology Conf. 1999, pp. 1266-1270.

[2] K. Sipilä, M. Jäsberg, J. Laiho-Steffens, and A. Wacker, "Soft handover gains in a fast power controlled WCDMA uplink," in Proc. IEEE Vehicular Technology Conf. 1999, pp. 1594-1598.

[3] R. D. Yates, "A framework for uplink power control in cellular radio systems," IEEE J. Sel. Areas Commun., vol. 13, no. 7, pp. 1341-1347, Sept. 1995.

[4] S. V. Hanly, "An algorithm for combined cell-site selection and power control to maximize cellular spread spectrum capacity," IEEE J. Sel. Areas Commun., vol. 13, no. 7, pp. 1332-1340, Sept. 1995.

[5] L. Mendo and J. M. Hernando, "On dimension reduction for the power control problem," IEEE Trans. Commun., vol. 49, no. 2, pp. 243-248, Feb. 2001.

[6] K. Hiltunen and M. Karlsson, "A novel WCDMA uplink capacity and coverage model including the impact of non-ideal fast power control and macro diversity," in Proc. IEEE Vehicular Technology Conf. 2003, pp. $98-102$.

[7] D. K. Kim and F. Adachi, "Theoretical analysis of reverse link capacity for an SIR-based power-controlled cellular CDMA system in a multipath fading environment," IEEE Trans. Veh. Technol., vol. 50, no. 2, pp. 452464, Mar. 2001.
[8] S. Dehghan, D. Lister, R. Owen, and P. Jones, "W-CDMA capacity and planning issues," IEE J. Electron. Commun. Engineering, vol. 12, no. 3, pp. 101-118, June 2000.

[9] A. Wacker, J. Laiho-Steffens, K. Sipilä, and M. Jäsberg, "Static simulator for studying WCDMA radio network planning issues," in Proc. IEEE Vehicular Technology Conf., pp. 2436-2440, 1999.

[10] J. Laiho, A. Wacker, and T. Novosad, Eds., Radio Network Planning and Optimisation for UMTS. John Wiley and Sons, 2001.

[11] L. Mendo and J. M. Hernando, "An efficient algorithm for determination of the optimum base-station assignment in cellular DS-CDMA systems," IEEE Trans. Commun., vol. 52, no. 3, pp. 435-445, Mar. 2004.

[12] W. Fleming, Functions of Several Variables, Second Ed. SpringerVerlag, 1977.

[13] K. T. Smith, Primer of Modern Analysis. Springer-Verlag, 1983.

[14] J. Zander, "Performance of optimum transmitter power control in cellular radio systems," IEEE Trans. Veh. Technol., vol. 41, no. 1, pp. 57-62, Feb. 1992.

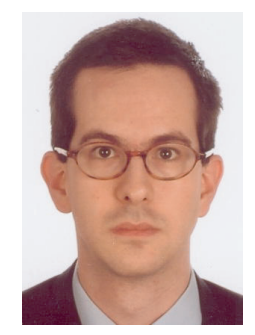

Luis Mendo was born in Madrid, Spain, on June 20, 1973. He received the M.Sc. and the Ph.D. degrees in telecommunication engineering from the Polytechnic University of Madrid, Madrid, Spain, in 1997 and 2001, respectively.

He has worked in radio network planning, and is currently with the Polytechnic University of Madrid. $\mathrm{He}$ is a coauthor of two textbooks on CDMA cellular systems, and has published several papers in the fields of CDMA system capacity and power control. His current research interests include CDMA networks, teletraffic, and Monte Carlo methods.

Dr. Mendo is a recipient of a national prize from the Telecommunication Engineering Professional Organization for his Ph.D. dissertation, and of two national awards for his M.Sc. thesis.

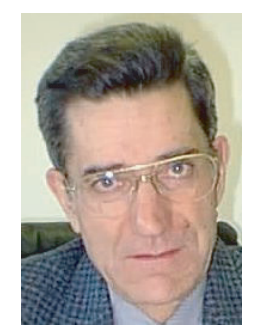

José M. Hernando (M'94) received the M.S. degree in communications engineering and the Ph.D. degree from the Polytechnic University of Madrid, Madrid, Spain, in 1967 and 1970, respectively.

Between 1967-1970, he was with ITT Laboratories of Spain doing research work in teletraffic theory. From 1970 until 1977, he was a Senior Engineer in the Communications Department of Iberia Airlines, Spain, working on planning, implementation, and maintenance of mobile radio networks, ground and air-to-ground in HF and VHF/UHF for both voice and data radiocommunications. In 1977, he joined the Signals, Systems and Radiocommunications Department, Polytechnic University of Madrid. Since then, he has been a Senior Professor devoted to educational and research work in radiocommunications. He has served as the Spanish Delegate in conferences and working groups in the Radiocommunications Sector of the International Telecommunications Union.

Dr. Hernando is member of the Spanish Bar of Telecommunications Engineers. 